\title{
CONVEX HULLS AND EXTREME POINTS OF SOME FAMILIES OF UNIVALENT FUNCTIONS(')
}

\author{
BY \\ D. J. HALLENBECK
}

\begin{abstract}
The closed convex hull and extreme points are obtained for the functions which are convex, starlike, and close-to-convex and in addition are real on $(-1,1)$. We also obtain this result for the functions which are convex in the direction of the imaginary axis and real on $(-1,1)$. Integral representations are given for the hulls of these families in terms of probability measures on suitable sets. We also obtain such a representation for the functions $f(z)$ analytic in the unit disk, normalized and satisfying $\operatorname{Re} f^{\prime}(z)>\alpha$ for $\alpha<1$. These results are used to solve extremal problems. For example, the upper bounds are determined for the coefficients of a function subordinate to some function satisfying $\operatorname{Re} f^{\prime}(z)>\alpha$.
\end{abstract}

Introduction. We shall determine the closed convex hulls and extreme points of some families of univalent functions. We utilize these results to solve specific extremal problems over certain of the families.

Let $\Delta$ denote the unit disk $\{z:|z|<1\}$ and let $A$ denote the set of functions analytic in $\Delta$. Then $A$ is known to be a locally convex linear topological space where the topology is given by uniform convergence on compact subsets of $\Delta[14$, p. 150]. We let $S$ denote the subset of $A$ consisting of the functions $f$ that are univalent in $\Delta$ and normalized so as to satisfy $f(0)=0$ and $f^{\prime}(0)=1$. Let $K, S t$ and $C$ denote the well-known subfamilies of $S$ which are respectively convex, starlike and close-to-convex. We will consider the subfamilies $K_{R}, S t_{R}$ and $C_{R}$ where for any class of functions $\exists$ we let $\Im_{R}=\{f: f \in \mathcal{\exists}$ and $f$ is real on $(-1$, 1) $\}$. We also consider the family of functions denoted by $F_{R}$ which are convex in the direction of the imaginary axis and real on $(-1,1)$. The functions in $F_{R}$ were studied by M. S. Robertson in [10]. Further we let $P(\alpha)$ denote the subfamily of $S$ consisting of those functions satisfying $\operatorname{Re} f^{\prime}(z)>\alpha$ where $0 \leq \alpha<1$.

The study of the convex hulls and extreme points of various families of univalent functions was initiated by L. Brickman, T. H. MacGregor, and D. R. Wilken in [2]. It was continued by the above authors and the present author in [1]. We shall use some of the basic results contained in [2]. $\& F$ shall denote the

Received by the editors December 1, 1972 and, in revised form, April 13, 1973.

AMS (MOS) subject classifications (1970). Primary 30A32, 30A40.

Key words and phrases. Univalent functions, starlike functions, starlike function with real coefficients, convex function, convex function with real coefficients, close-to-convex function, closeto-convex function with real coefficients, extreme point, integral representation, probability measures, subordination, continuous linear functional.

(1) Some of the results contained herein formed a portion of the author's Ph.D. dissertation prepared under the direction of Professor Thomas H. MacGregor.

Copyright $\odot$ 1974, American Mathematical Society 
closed convex hull of a family of functions $F$. $E \& F$ shall denote the set of extreme points of $\mathscr{H F}$. We recall that whenever $F$ is compact, $\varepsilon \not F \subset F$.

We find that $\mathcal{H} K_{R}$ consists of the functions represented as

$$
f(z)=\int_{x} \frac{1}{x-\bar{x}} \log \frac{1-\bar{x} z}{1-x z} d \mu(x)
$$

where $\mu$ varies over the probability measures on $X=\{x:|x|=1$ and $\operatorname{Im} x$ $\geq 0\}$. We show that $\& K_{R}=\& F_{R}$. We find further that $\& S t_{R}=\& C_{R}=\& S_{R}$ where $\& S_{R}$ was determined in [2, p. 95]. Finally we prove that $\mathcal{H P}(\alpha)$ consists of the functions represented as

$$
f(z)=(2 \alpha-1) z+(2 \alpha-2) \int_{X} \bar{x} \log (1-x z) d \mu(x)
$$

where $\mu$ varies over the probability measures on the unit circle $X$.

We recall the definition of subordination between two functions $f$ and $g$ which are both analytic in $\Delta$. We say that $f$ is subordinate to $g$, denoted by $f \prec g$, if there exists an analytic function $\phi(z)$ so that $\phi(0)=0,|\phi(z)|<1$ and $f(z)$ $=g(\phi(z))$ for $z$ in $\Delta$. We prove that if $f<F$ where $F \in P(\alpha)$ and if $f(z)$ $=\sum_{n=1}^{\infty} a_{n} z^{n}$ then $\left|a_{n}\right| \leq 1$ for $n=1,2,3, \ldots$. We also show that if $f \in P(\alpha)$ then

$$
f(z) / z<(2 \alpha-1)+(2 \alpha-2) \log (1-z) / z .
$$

As a consequence we show that if $f \in P(\alpha)$ then

$$
\operatorname{Re}(f(z) / z) \geq(2-2 \alpha)(1 /|z|) \log (1+|z|)+(2 \alpha-1)
$$

for all $z$ in $\Delta$. We also determine the radius of convexity of $P(1 / 2)$ to be $1 / \sqrt{2}$. This result has also recently been announced by $D$. Shaffer [13].

1. The convex hulls and extreme points of $K_{R}, S t_{R}, C_{R}$ and $F_{R}$.

Theorem 1. Let $X$ be $\{z:|z|=1, \operatorname{Im} z \geq 0\}$, let $\mathbb{P}$ be the set of probability measures on $X$, and let $\Im$ be the set of functions $f_{\mu}$ on $\Delta$ defined by

$$
f_{\mu}(z)=\int_{X} \frac{z}{(1-x z)(1-\bar{x} z)} d \mu(z), \quad \mu \in \mathscr{P} .
$$

Then $\mathcal{H} S t_{R}=\mathcal{H} C_{R}=9$, the map $\mu \rightarrow f_{\mu}$ is one-to-one, and the extreme points of $\mathcal{H} t_{R}$ and $\& C_{R}$ are precisely the functions $z /(1-x z)(1-\bar{x} z), x \in X$.

Proof. Since $z /(1-x z)(1-\bar{x} z)$ is in $S t_{R}$ and $S t_{R} \subset C_{R}$ we have as a consequence of [2, Theorem 1, p. 93] that $\exists \subset \& S t_{R} \subset \& C_{R}$. If $f \in S t_{R}$ then $f \in S_{R}$. In [2] it was proven that $S_{R} \subset \mathcal{G}$. Hence, $S t_{R} \subset C_{R} \subset \exists$ which implies that $\mathcal{H} S t_{R} \subset \mathscr{H} C_{R} \subset \exists$ since $\exists$ is convex and compact.

Hence $\psi S S t_{R}=\alpha C_{R}=$ 7. The map $\mu \rightarrow f_{\mu}$ is known [2] to be one-to-one and consequently the remark concerning the extreme points follows from $[2$, Theorem 1]. 
Remarks. It is easy to show that $S t_{R} \subseteq C_{R} \subseteq S_{R}$. In [2, Theorem 4] it was shown that $\mathcal{H} S_{R}=\Im$ where $\Im$ is defined as above. Hence we may conclude that $\mathcal{H} S t_{R}=\& C_{R}=\& S_{R}$. Of course it follows that each of these hulls have the same extreme points. We remark that $\Im=T$, the set of typically real functions $[2$, p. 95].

Theorem 2. Let $X$ be $\{z:|z|=1, \operatorname{Im} z \geq 0\}$, let $\mathscr{P}$ be the set of probability measures on $X$ and let $\exists$ be the set of all functions $f_{\mu}$ defined on $\Delta$ by

$$
f_{\mu}(z)=\int_{X} \frac{1}{x-\bar{x}} \log \frac{1-\bar{x} z}{1-x z} d \mu(x), \quad \mu \in \mathscr{P} .
$$

Then $\mathcal{H} K_{R}=9$, the map $\mu \rightarrow f_{\mu}$ is one-to-one, and the extreme points of $\mathcal{H} K_{R}$ are precisely the functions $(x-\bar{x})^{-1} \log ((1-\bar{x} z) /(1-x z))$ for $|x|=1$.

Proof. The operator $L$ defined by $(L f)(z)=\int_{0}^{z}(f(\omega) / \omega) d \omega$ is known to be a linear homeomorphism of the space of analytic functions on $\Delta$ that vanish at 0 and further that $L(S t)=K$. Since the operator $L$ also preserves real coefficients, it follows that $L\left(S t_{R}\right)=K_{R}$. We note that since

$$
L\left(\frac{z}{(1-x z)(1-\bar{x} z)}\right)=\frac{1}{x-\bar{x}} \log \frac{1-\bar{x} z}{1-x z}
$$

it follows by Theorem 1 that $L\left(\& S t_{R}\right)=\alpha K_{R}=9$. The fact that the map $\mu \rightarrow f_{\mu}$ is one-to-one follows from the previous theorem since the operator does not alter this condition. Hence the conclusion concerning the extreme points follows from [2, Theorem 1].

Remarks. The kernel function $K(z, x)=(x-\bar{x})^{-1} \log ((1-\bar{x} z) /(1-x z))$ can be defined continuously at $x=1,-1$ and indeed $K(z, 1)=z /(1-z)$ and $K(z,-1)=z /(1+z)$.

Theorem 3. Let $X$ be $\{z:|z|=1, \operatorname{Im} z \geq 0\}$, let $\mathfrak{P}$ be the set of probability measures on $X$ and let $\Im$ be the set of all functions $f_{\mu}$ defined on $\Delta$ by

$$
f_{\mu}(z)=\int_{X} \frac{1}{x-\bar{x}} \log \frac{1-\bar{x} z}{1-x z} d \mu(x), \quad \mu \in \mathscr{P} .
$$

Then $\mathcal{H} F_{R}=\exists$, the map $\mu \rightarrow f_{\mu}$ is one-to-one, and the extreme points of $\mathcal{H} F_{R}$ are precisely the functions $(x-\bar{x})^{-1} \log ((1-\bar{x} z) /(1-x z))$ for $|x|=1$.

Proof. It is an equivalent form of a result of M. S. Robertson [11] that if $f \in F_{R}$ then

$$
f(z)=\int_{X} \frac{1}{x-\bar{x}} \log \frac{1-\bar{x} z}{1-x z} d \mu(x) \quad \text { where } \mu \in \mathcal{P} .
$$

Hence $F_{R} \subset \exists$ and consequently $\mathscr{H} F_{R} \subset \exists$ since $\exists$ is closed and convex. However, each function $(x-\bar{x})^{-1} \log ((1-\bar{x} z) /(1-x z))$ is real on $(-1,1)$ and convex by the previous theorem, since $\varepsilon \not K_{R} \subset K_{R}$. These functions can also be 
seen to be convex by a simple direct computation. Hence each kernel function $K(z, x)=(x-\bar{x})^{-1} \log ((1-\bar{x} z) /(1-x z))$ is in $F_{R}$. Consequently by [2, Theorem 1], we conclude that $\exists \subset \mathcal{H} F_{R}$. Therefore $\mathcal{H} F_{R}=\exists$. By Theorem 2 the mapping $\mu \rightarrow f_{\mu}$ is known to be one-to-one and so the conclusion about the extreme points follows from Theorem 1 of [2].

Remarks. It is easy to show that $K_{R} \subsetneq F_{R}$. However, by the results of Theorems 2 and 3, we see that $\mathcal{H} K_{R}=\mathcal{H} F_{R}$ and these hulls have the same extreme points.

2. The convex hulls and extreme points of the class $P(\alpha)$. We recall that $P(\alpha)$ consists of those functions satisfying $f(0)=0, f^{\prime}(0)=1$, and $\operatorname{Re} f^{\prime}(z)>\alpha$ for $z$ in $\Delta$ where $0 \leq \alpha<1$. We remark that the third condition is known [8] to imply that $f(z)$ is one-to-one and so given our normalizations we have $P(\alpha)$ a compact subfamily of $S$. The class $P(0)$ was investigated by T. H. MacGregor in [4]. We remark that every theorem dealing with $P(\alpha)$ contained in this paper is valid for $\alpha<1$ with the single exception of Theorem 5. We note that if $\alpha<0$ then the derivative of functions in $P(\alpha)$ can have a zero in $\Delta$, so that $P(\alpha) \oplus S$.

Theorem 4. Let $X$ be $\{z:|z|=1\}$, let $\mathbb{P}$ be the set of probability measures on $X$ and let $\Im$ be the set of functions $f_{\mu}$ on $\Delta$ defined by

$$
f_{\mu}(z)=(2 \alpha-1) z+(2 \alpha-2) \int_{X} \bar{x} \log (1-x z) d \mu(x), \quad \mu \in \mathfrak{P} .
$$

Then $\mathcal{H} P(\alpha)=P(\alpha)=\exists$, the map $\mu \rightarrow f_{\mu}$ is one-to-one, and the extreme points of $\mathcal{H} P(\alpha)$ are precisely the functions $(2 \alpha-1) z+(2 \alpha-2) \bar{x} \log (1-x z), x \in X$.

Proof. Since $\operatorname{Re} f^{\prime}(z)>\alpha$ for $z$ in $\Delta$ and $f^{\prime}(0)=1$, it follows by applying the Herglotz formula to $\left(f^{\prime}(z)-\alpha\right) /(1-\alpha)$ that

$$
f^{\prime}(z)=\int_{X} \frac{1+(1-2 \alpha) x z}{1-x z} d \mu(x) \quad \text { for some } \mu \in \mathfrak{P} .
$$

Hence by using $f(0)=0$ we conclude through integration that

$$
f(z)=(2 \alpha-1) z+(2 \alpha-2) \int_{X} \bar{x} \log (1-x z) d \mu(x) .
$$

Hence $P(\alpha) \subset \exists$ and so $\mathcal{H} P(\alpha) \subset \not \mathcal{Y}=\Im$. The statement $\mathcal{H} P(\alpha)=P(\alpha)$ is clear since $P(\alpha)$ is a closed convex set. It is easy to verify that the kernel function

$$
K(z, x)=(2 \alpha-1) z+(2 \alpha-2) \bar{x} \log (1-x z)
$$

is an element of $P(\alpha)$ for each $x \in X$. Hence by [2, Theorem 1] it follows that $\exists \subset \mathcal{H}(\alpha)$. Therefore $\mathcal{H} P(\alpha)=P(\alpha)=\Im$. The mapping $\mu \rightarrow f_{\mu}$ is easily seen to be one-to-one since this property is known to hold for the Herglotz representation $[9$, p. 30].

The assertion about the extreme points follows in the usual way from [2, Theorem 1]. 
Remarks. We recall the definition of the class of functions which are convex of order $\alpha$ which we denote by $K(\alpha)$. A function $f$ is in $K(\alpha)$ if and only if $\operatorname{Re}\left(1+z f^{\prime \prime}(z) / f^{\prime}(z)\right)>\alpha$ for $z$ in $\Delta$ where $0 \leq \alpha<1$ and $f$ has the same normalizations as $S$. These compact subfamilies of $S$ were introduced by M. S. Robertson in [10]. In [1] the set $\mathcal{H K}(\alpha)$ was determined exactly for all $\alpha<1$. When $\alpha=1 / 2$ it was shown that

$$
\mathcal{H} K(1 / 2)=\left\{\int_{X}-\bar{x} \log (1-x z) d \mu(x): \mu \in \mathscr{P}\right\}
$$

where $X$ is the unit circle. We see from Theorem 4 above that $\mathscr{H} K(1 / 2)$ $=\mathcal{H}(1 / 2)=P(1 / 2)$. This is of some interest since $K(1 / 2) \subseteq P(1 / 2)$ is a direct implication of our Theorem 7 and the foregoing remark given that $K(1 / 2) \subset \& K(1 / 2)$.

Corollary 4.1. If $f(z)=z+\sum_{n=2}^{\infty} a_{n} z^{n} \in P(\alpha)$, then $\left|a_{n}\right| \leq(2-2 \alpha) / n$ for $n=2,3, \ldots$.

Proof. By Theorem 4 the extreme points of $P(\alpha)$ are given by $(2 \alpha-1) z$ $+(2 \alpha-2) \bar{x} \log (1-x z), x \in X$. It is easy to verify that the $n$th coefficient in the power series expansion for the kernel function is bounded in modulus by $(2-2 \alpha) / n$. Therefore, the same bound will hold for each function in $P(\alpha)$.

Remarks. This corollary could easily have been proven without knowledge of the extreme points by using the known bound on the modulus of the $n$th coefficient in the power series expansion of a function $p$ satisfying $\operatorname{Re} p(z)>\alpha$ for $z$ in $\Delta$. We include it because of its relevance to the next theorem.

The next theorem depends upon a fact proven by T. H. MacGregor in [6]. Suppose $G$ is a compact subset of $A$ and $F$ is the class of all functions subordinate to each function in $G$. Let $F_{0}$ be the subset of $F$ of functions subordinate to some function in $\varepsilon \mathscr{H} G$. If $J$ is a complex-valued, continuous, linear functional on $A$, then

$$
\max _{f \in F}|J(f)|=\max _{f \in F_{0}}|J(f)| .
$$

We also need a result of W. Rogozinski $[12$, p. 64] which we state now. Suppose that $f(z)=\sum_{n=1}^{\infty} a_{n} z^{n}$ is subordinate to $F(z)=\sum_{n=1}^{\infty} A_{n} z^{n}$ in $\Delta$. If, for $1 \leq k$ $\leq n$, the numbers $A_{k}$ are nonnegative, nonincreasing, and convex, then $\left|a_{n}\right|$ $\leq A_{1}$, for $k=1,2, \ldots, n$.

Theorem 5. Let $f(z)=\sum_{n=1}^{\infty} a_{n} z^{n}$ be analytic in $\Delta$ and be subordinate to some function in $P(\alpha)$ where $0 \leq \alpha<1$. Then $\left|a_{n}\right| \leq 1$ for $n=1,2, \ldots$.

Proof. Since the family $P(\alpha)$ is compact the arguments referred to above by $T$. H. MacGregor given in [6] show that in order to maximize $\left|a_{n}\right|$ we need only consider the functions $f$ which are subordinate to a function in $\varepsilon \mathcal{H} P(\alpha)$. Hence, by Theorem $4, f(z)$ has the form

$$
f(z)=(2 \alpha-1) \phi(z)+(2 \alpha-2) \bar{x} \log (1-x \phi(z)),
$$


where $|x|=1, \phi$ is analytic for $|z|<1,|\phi(z)|<1$, and $\phi(0)=0$. The function $\phi(z) / x$ has the same properties as $\phi$ and a function $f$ and $x f$ have $n$th coefficients with the same modulus. Hence we may assume $x=1$, that is, $f$ is subordinate to $F(z)=(2 \alpha-1) z+(2 \alpha-2) \log (1-z)$. Let $F(z)=\sum_{n=1}^{\infty} A_{n} z^{n}$. Then $A_{1}=1$ and $A_{n}=(2-2 \alpha) / n$ for $n=2,3, \ldots$. Since $0 \leq \alpha<1$ the sequence $\left\{A_{n}\right\}$ is seen to consist of nonnegative real numbers. Since

$$
A_{n+1}-A_{n}=-(2-2 \alpha) / n(n+1)
$$

the sequence is seen to be nonincreasing. Finally, since we have

$$
A_{n}-2 A_{n+1}+A_{n+2}=(2-2 \alpha) \frac{2}{n(n+1)(n+2)}
$$

we conclude that the sequence $\left\{A_{n}\right\}$ is also convex. Hence by a direct application of Rogozinski's result mentioned above we conclude $\left|a_{n}\right| \leq 1$ for $n=2,3, \ldots$.

Remarks. This result can be seen to be sharp for each $n$ by choosing $\phi(z)=z^{n}$.

Before we prove our next theorem it is appropriate to introduce a result proven by R. J. Libera in [3] which we need for our proof.

Lemma 1. Let $f$ be analytic and univalent for $|z|<1$. Suppose $f(0)=0$ and $f(\Delta)$ is convex. If $g(z)=(1 / z) \int_{0}^{z} f(\omega) d \omega$ then $g$ is also univalent and convex for $|z|<1$.

Using Libera's result above, we prove the following simple lemma.

Lemma 2. The function $\log (1-z) / z$ is univalent and convex for $z$ in $\Delta$.

Proof. We may write

$$
1+\frac{\log (1-z)}{z}=\frac{1}{z} \int_{0}^{z}\left(1+\frac{-1}{1-\omega}\right) d \omega .
$$

The function $f(z)=1+1 /(1-z)$ is univalent and convex for $z$ in $\Delta$. It also satisfies $f(0)=0$. Consequently, by Lemma 1 above we conclude that 1 $+\log (1-z) / z$ is univalent and convex for $z$ in $\Delta$ and hence $\log (1-z) / z$ also has these same properties.

Theorem 6. If $f(z) \in P(\alpha)$ then

$$
f(z) / z \prec(2 \alpha-1)+(2 \alpha-2) \log (1-z) / z \text { for } z \text { in } \Delta .
$$

Proof. We know by Theorem 4 that

$$
\frac{f(z)}{z}=(2 \alpha-1)+(2 \alpha-2) \int_{x} \frac{\log (1-x z)}{x z} d \mu(x)
$$

where $\mu$ is a probability measure on $X$, the unit circle. By the previous lemma we know that $\log (1-z) / z$ is univalent and convex for $|z|<1$. The result follows directly. 
Corollary 6.1. If $f(z) \in P(\alpha)$, then

$$
\operatorname{Re}(f(z) / z) \geq(2 \alpha-1)+(2-2 \alpha)(1 /|z|) \log (1+|z|) \text { for }|z|<1 \text {. }
$$

Proof. By Theorem 6 we know that

$$
f(z) / z \prec g(z)=(2 \alpha-1)+(2 \alpha-2) \log (1-z) / z
$$

for $|z|<1$. The function $g(z)$ is univalent and convex by Lemma 2 . It follows by the convexity, conformality, and the realness of $g$ that the image of the disk $|z| \leq r$ for any $0 \leq r<1$ has a support line at $(2 \alpha-1)+(2-2 \alpha)(1 /|z|)$ $\cdot \log (1+|z|)$ which is perpendicular to the real axis at that point. It is clear that the image must lie wholly to the right of this line. Since the image of $|z| \leq r$ under $f(z) / z$ is contained in the image of $|z| \leq r$ under $g(z)$ the result follows.

Remarks. 1 . This result in the case $\alpha=0$ was proven by T. H. MacGregor in $[4$, p. 533].

2. An easy argument shows that equality holds only for the kernel functions $(2 \alpha-1) z+(2-2 \alpha) \bar{x} \log (1-x z), x \in X$.

3. It is a problem in calculus to show that $(1 /|z|) \log (1+|z|)>\log 2$ for $z$ in $\Delta$. Hence if $f \in P(\alpha)$ we see that $\operatorname{Re}(f(z) / z)>(2 \alpha-1)+(2-2 \alpha) \log 2$.

4. A proof of the corollary independent of knowledge of extreme points can be fashioned by appropriately integrating the known lower bound on the real part of the derivative of a function in $P(\alpha)$.

The following result as mentioned earlier has also seen announced by $D$. Shaffer [13].

Theorem 7. The radius of convexity of $P(1 / 2)$ is $1 / \sqrt{2}$.

Proof. If $g(z)=z f^{\prime}(z)$, then $g(z)$ is starlike if and only if $f(z)$ is convex. Note that $\operatorname{Re}(g(z) / z)=\operatorname{Re} f^{\prime}(z)>1 / 2$ for $z$ in $\Delta$. The radius of starlikeness of the class of functions satisfying the $g(z) / z>1 / 2$ was found by T. H. MacGregor in $[5$, p. 75] to be $1 / \sqrt{2}$. Hence the radius for $P(1 / 2)$ is $1 / \sqrt{2}$.

Remarks. The radius of convexity of $P(0)$ was determined by T. H. MacGregor in $[4$, p. 33] to be $\sqrt{2}-1$. The problem of determining the radius of convexity for arbitrary $\alpha$ and the problem of determining the radius of starlikeness are, as far as we can determine, open.

Acknowledgement. The author wishes to thank the referee for his useful suggestions.

\section{REFERENCES}

1. L. Brickman, D. J. Hallenbeck, T. H. MacGregor and D. R. Wilken, Convex hulls and extreme points of families of starlike and convex mappings, Trans. Amer. Math. Soc. (to appear).

2. L. Brickman, T. H. MacGregor and D. R. Wilken, Convex hulls of some classical families of univalent functions, Trans. Amer. Math. Soc. 156 (1971), 91-107. MR 43 \#494. 
3. R. J. Libera, Some classes of regular univalent functions, Proc. Amer. Math. Soc. 16 (1965), 755-758. MR 31 \#2389.

4. T. H. MacGregor, Functions whose derivative has a positive real part, Trans. Amer. Math. Soc. 104 (1962), 532-537. MR 25 \#4090.

5. The radius of convexity for starlike functions of order 1/2, Proc. Amer. Math. Soc. 14 (1963), 71-76. MR 27 \#283.

6. - Applications of extreme-point theory to univalent functions, Michigan Math. J. 19 (1972), 361-376.

7. Z. Nehari, Conformal mapping, McGraw-Hill, New York, 1952. MR 13, 640.

8. K. Noshiro, On the theory of schlicht functions, Journal of the Faculty of Science, Hokkaido Imperial University, Sapporo (1934/35), pp. 129-155.

9. P. Porcelli, Linear spaces of analytic functions, Rand McNally, Chicago, Ill., 1966. MR 41 \#4219. 10. M. S. Robertson, On the theory of univalent functions, Amer. J. Math. (2) 37 (1936), 374-408.

11.—, Analytic functions star-like in one direction, Amer. J. Math. 58 (1936), 465-472.

12. W. Rogozinski, On the coefficients of subordinate functions, Proc. London Math. Soc. (2) 48 (1943), 48-82. MR 5, 36.

13. D. B. Shaffer, Analytic functions $f(z)$ with $\operatorname{Re} f(z)>1 / 2$ and applications, Notices Amer. Math. Soc. 19 (1972), A704-A705. Abstract \# 727-B287.

14. A. E. Taylor, Introduction to functional analysis, Wiley, New York; Chapman \& Hall, London, 1958. MR 20 \#5411.

Department of Mathematics, Universtty of Delaware, Newark, Delaware 19711 